\title{
Was kann unser Genom uns noch erzählen?
}

$\mathrm{K}$ rebs ist nach unserem heutigen Verständnis eine genetische Erkrankung. Krebsverursachende genetische Defekte im und am Erbgut von Körperzellen werden im Laufe des Lebens erworben, sind in manchen Fällen auch ein erblicher Bestandteil des $\mathrm{Ge}-$ noms. Am Horizont unserer Erkenntnisse in diesem Bereich kündigt sich die Möglichkeit an, solche genetischen Defekte nicht nur anhand der anamnestischen Datenlage zu vermuten, oder aus der bisher möglichen Vogelperspektive des Karyotyps und gezielter molekularer Einzeluntersuchungen mit einer bestimmten Wahrscheinlichkeit anzunehmen, sondern im Detail durch Hochdurchsatz-Sequenzierung des gesamten Genoms zu ermitteln. Bevor solche Untersuchungen allerdings für die Patientenbehandlung informativ sind, ist eine Kartographierungsphase unerhörten Ausmaßes erforderlich. Dort wird man zwar bald einige niedrig hängende Früchte der Erkenntnis pflücken können, jedoch gibt es eine beträchtliche Komplexität der Veränderungen. In den bisher erhältlichen Untersuchungen wurde gerade kürzlich wieder im Lungenkarzinom und im Melanom eine erhebliche ,Vernarbung" des Genoms solcher Zellen selbst im Vergleich zu normalen Zellen der gleichen Person - mit zig bis zu tausenden kleinerer und größerer Defekte gezeigt. Die wirkliche Ernte dieser Arbeit wird erfordern, die Daten von Hunderten Fällen einer Diagnose miteinander $\mathrm{zu}$ vergleichen, um $\mathrm{zu}$ verstehen, welche Signalkaskaden regelhaft von bestimmten Veränderungen betroffen sind, und auch, welche morphologisch völlig unterschiedlichen Tumorerkrankungen vielleicht molekulare und damit behandlungsstrategische Ähnlichkeiten aufweisen, die sich bei mikroskopischer Betrachtung nicht erschlossen haben. Das internationale ICGC Konsortium hat begonnen, sich den ersten Schritten dieser
Fragestellung in vorbildlicher Weise anzunehmen.

Dieser Weg wird schwer, aufregend, ja geradezu spannend, und am Ende hoffentlich so erfolgreich wie viele von uns sich das erträumen. Bereits jetzt kommen jeden Tag neue Erkenntnisse zu unserem Verständnis hinzu, und einige davon sind auch für Prävention und personalisierte Therapie bereits heute von erheblicher Bedeutung. Gerade in diesen Zeiten des Aufbruchs ist eine fundierte, expertenkontrollierte und verständliche humangenetische Beratung unserer Patienten zur individuellen Risikoeinschätzung und möglichen Differentialdiagnose rarer Tumordispositionssyndrome notwendig.

Höchste Zeit also für Onkopipeline, mit Ihnen gemeinsam einmal einen genauen Blick auf den Stand der Dinge genetischer Beratung zu werfen, meint

Ihr Christof Kalle 